\title{
Tumor de Frantz-Gruber, un tumor sólido quístico pseudopapilar del páncreas infrecuente
}

\author{
Frantz-Gruber tumor, an infrequent solid \\ pseudopapillary tumor of the pancreas
}
Frederick Miranda-Castillo, ${ }^{*}$ Danna Auria-Aspiazu, ${ }^{*}$ Jenniffer Plaza-Vélez, Margarita Villacís-Muñoz, ${ }^{\ddagger}$ Carlos Benites-Ordinola ${ }^{\S}$

Palabras clave: Tumor de páncreas, tumor pseudopapilar, tumor de FrantzGruber.

Keywords: Pancreatic tumor, pseudopapillary tumor, Frantz-Gruber

tumor.

\footnotetext{
" Médico Postgradista.

¥ Médico Cirujano

Oncólogo.

$\S$ Médico Cirujano

General.

Servicio de Cirugía General del Instituto Oncológico Nacional "Dr. Juan Tanca Marengo" - ION SOLCA. Guayaquil, Ecuador.
}

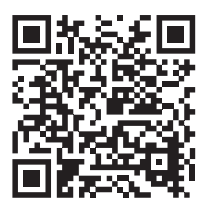

\section{RESUMEN}

Introducción: El tumor pseudopapilar sólido-quístico o tumor de Frantz-Gruber es una neoplasia de páncreas poco frecuente, con mayor presentación en el sexo femenino en las primeras décadas de vida, con sintomatología inespecífica y características histopatológicas definidas. Objetivo: Reportar un caso de un tumor sólido quístico pseudopapilar localizado en la cabeza del páncreas en una paciente joven. Presentación del caso: Paciente femenino con diagnóstico de tumor de Frantz-Gruber en un sitio infrecuente del páncreas, además de la revisión de su epidemiología, presentación clínica e histopatológica, tratamiento y pronóstico. Conclusión: Es una neoplasia rara que se presenta en menos de $1 \%$, con comportamiento benigno y con bajas probabilidades de malignización si su resección es completa.

\section{ABSTRACT}

Introduction: Solid-cystic pseudopapillary tumor or Frantz-Gruber tumor is a rare pancreas neoplasm, with a higher presentation in females in the first decades of life, with nonspecific symptoms and definite histopathological characteristics. Objective: To report a rare case of a solid pseudopapillary cystic tumor located in the head of the pancreas in a young patient. Case presentation: Presentation of a clinical case of a patient diagnosed with a Frantz-Gruber tumor in an uncommon site of the pancreas; besides the review of its epidemiology, clinical and histopathological presentation, treatment and prognosis. Conclusion: It is a rare neoplasm that occur in less than $1 \%$, with benign behavior and with low probabilities of malignancy if its resection is complete.

\section{INTRODUCCIÓN}

$\mathrm{E}^{\prime}$ I tumor pseudopapilar sólido, quístico del páncreas es una entidad poco frecuente, representando menos de $1 \%$ en la incidencia de todas las neoplasias pancreáticas. ${ }^{1}$

Esta neoplasia también llamada tumor de Frantz-Gruber fue descrita por primera vez en 1959. ${ }^{2-4}$ Histológicamente se caracteriza por una mezcla de áreas sólidas con pseudoquistes y estructuras pseudopapilares y hemorrágicas, red microvascular que forma pseudorrosetas y presencia de células eosinófilas o espumosas. ${ }^{5} \mathrm{Su}$ incidencia es en mujeres jóvenes entre la segunda y cuarta décadas de vida. ${ }^{1-3}$

Se considera de bajo grado de malignidad, ya que alrededor de 10 a 15\% desarrollan enfermedad metastásica. ${ }^{1-4}$ Preoperatoriamente no es fácil distinguirlo de otros tumores quísticos del páncreas, en muchas ocasiones se da como hallazgo radiológico y en otras puede ser diagnosticado erróneamente como pseudoquiste pancreático. ${ }^{2}$

\section{PRESENTACIÓN DEL CASO}

Paciente femenino de 13 años de edad sin antecedentes patológicos personales o quirúr-

Citar como: Miranda-Castillo F, Auria-Aspiazu D, Plaza-Vélez J, Villacís-Muñoz M, Benites-Ordinola C. Tumor de FrantzGruber, un tumor sólido quístico pseudopapilar del páncreas infrecuente. Cir Gen. 2020; 42 (3): 223-227. https://dx.doi. org/10.35366/99964 
gicos de importancia, es traída a consulta por su madre con cuadro clínico de cuatro meses de evolución caracterizado por presentar plenitud postprandial y masa palpable en región epigástrica, sin pérdida de peso ni fiebre. Al examen físico la paciente mostraba distensión abdominal leve, dolor a la palpación profunda en región de epigastrio con masa palpable a este nivel de aproximadamente $4 \mathrm{~cm}$, sin adenopatías ni datos de irritación peritoneal.

Análisis sanguíneos como biometría hemática completa, química sanguínea, entre éstos los de interés (amilasa y lipasa), uroanálisis se reportan dentro de parámetros normales.

Se realizan estudios de imagen: ecografía abdominopélvica compatible con lesión tumoral heterogénea retroperitoneal con áreas quísticas en la vecindad de la cabeza del páncreas que mide $6 \times 10.1 \mathrm{~cm}$ por encima del área suprarrenal; tomografía computarizada (TC) de abdomen y pelvis simple y con contraste endovenoso, se evidencia lesión tumoral heterogénea tabicada con captación del contraste en la periferia y tabiques, presencia de áreas de menor densidad quísticas localizada en la cabeza del páncreas de contornos bien delimitados que miden $5.9 \times 6.7 \times 7 \mathrm{~cm}$; cuerpo y cola pancreática sin alteraciones, sin dilatación del conducto de Wirsung, sin presencia de obliteración de la grasa perivascular en arteria mesentérica superior ni del eje esplenoportal, ni evidencia de adenomegalias retroperitoneales (Figura 1).

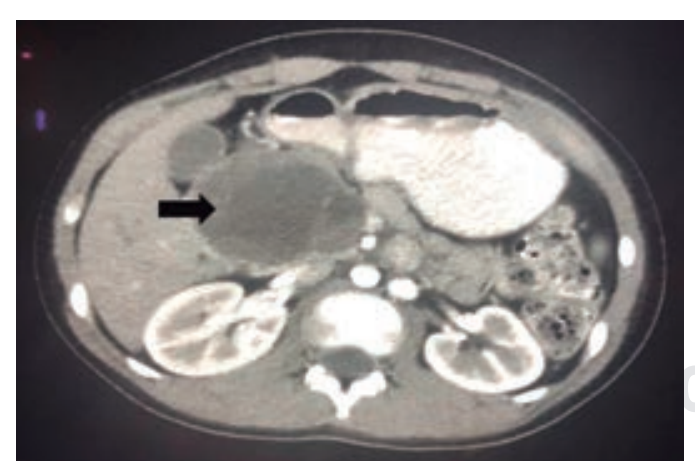

Figura 1: Tomografía axial computarizada con contraste oral y endovenoso donde se evidencia masa tumoral heterogénea tabicada (flecha negra), con áreas de menor densidad quistica localizadas en cabeza del páncreas, contornos bien delimitados $(5.9 \times 6.7 \times 7 \mathrm{~cm})$.

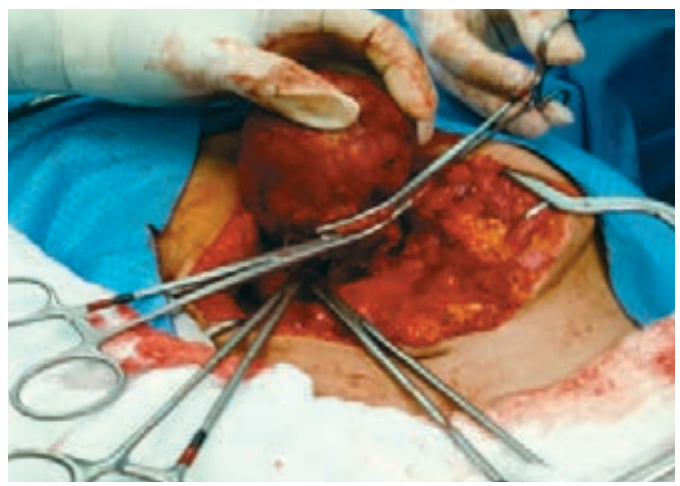

Figura 2: Enucleación de masa tumoral dependiente de cabeza del páncreas.

La paciente fue sometida a laparotomía exploradora donde se identifica masa tumoral de aproximadamente $6 \times 8 \mathrm{~cm}$ a nivel de la cabeza del páncreas adosada a la segunda porción del duodeno, por lo que se procede a realizar enucleación por medio de disección de segunda porción de duodeno y porción transpancreática involucrando conducto pancreático principal, se realiza tutorización del mismo con sonda Nélaton № 12, exteriorizándola a través de segunda porción del duodeno hacia la pared abdominal a la altura del flanco derecho, siendo fijada con sutura de polidioxanona PDX 3.0 (Figuras 2 a 4).

La paciente cursó un postoperatorio favorable de 33 días, además de las medidas generales se implementó antibioticoterapia de amplio espectro correspondiente a amikacina $680 \mathrm{mg}$ cada día intravenoso por 10 días acompañado de imipenem 500 mg cada seis horas intravenoso por 15 días, para pasar luego a cefalexina $500 \mathrm{mg}$ por vía oral cada ocho horas por siete días y posterior al alta, cefuroxima 500 mg por vía oral cada 12 horas por cinco días; nutrición parenteral iniciando al tercer día postquirúrgico y manteniéndose por 10 días, al séptimo día postquirúrgico se inició dieta líquida estricta para luego progresarla a dieta blanda que fue bien tolerada y el uso de análogo de somatostatina desde el inicio del postquirúrgico a razón de $4 \mu \mathrm{g}$ intravenoso cada ocho horas hasta su alta, además de establecerse un control riguroso del dren, realizando el clampleo diario de éste hasta cumplir las 24 horas para luego continuar con el control fistulográfico, mismo 
que no reportó un stop o fugas, por lo que se decidió el retiro del dren sin mostrar alteración alguna (Figura 5).

El resultado histopatológico reveló positividad para carcinoma papilar sólido pancreático con ausencia de malignidad en tejidos circundantes como epiplón mayor y ganglio linfático pancreático (Figura 6).

\section{DISCUSIÓN}

El tumor pseudopapilar sólido quístico de páncreas es una neoplasia poco frecuente, en nuestro instituto representa menos de $1 \%$ del total de neoplasias pancreáticas, porcentaje discretamente inferior a lo reportado en la literatura mundial. ${ }^{1-3}$ En 95\% suele aparecer en mujeres jóvenes con una media de edad de 20 años; sin embargo, en nuestro caso se presentó a una edad muy temprana. ${ }^{6}$ Los tumores pueden localizarse a cualquier nivel en la glándula pancreática, aunque aparecen con más frecuencia en el cuerpo y cola del páncreas, a diferencia de la presentación de nuestro caso, el cual fue en la cabeza del páncreas. Aunque la mayoría de estos tumores tienen comportamiento benigno con una larga tasa de sobrevida, pueden llegar a generar metástasis hasta en 15\% aproximadamente; el reporte histopatológico que hace referencia a presencia de invasión perineural nos hace pensar en una posible malignización de la lesión, lo que hace imprescindible el seguimiento de esta paciente. . $^{1,6,7}$

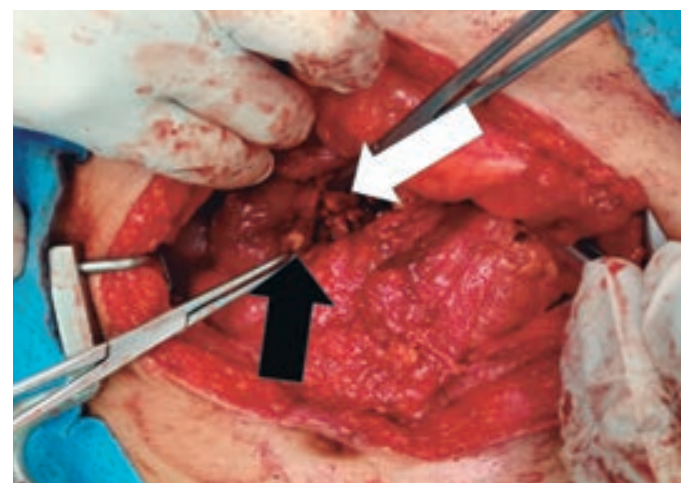

Figura 3: Porción transpancréatica que involucra conducto pacréatico principal extremo proximal (flecha blanca), extremo distal (flecha negra).

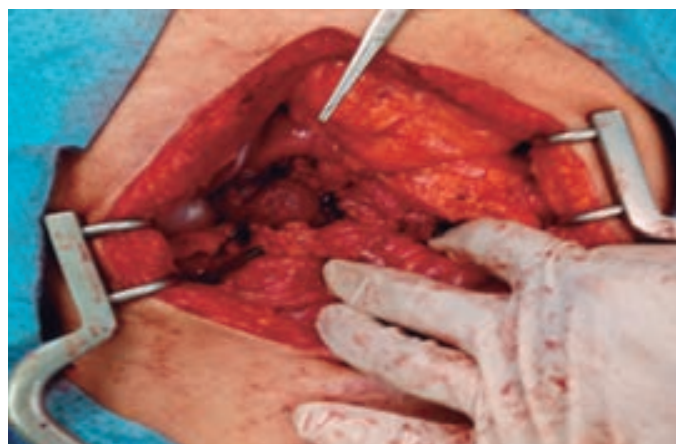

Figura 4: Tutorización del conducto pancreático con sonda Nélaton $N^{\circ} 12$.

Clínicamente los pacientes muestran dolor abdominal, masa palpable y dispepsia, similar al cuadro clínico presentado en nuestro caso, a pesar de que la literatura refiere al diagnóstico incidental como la mayor forma de presentación. ${ }^{1-3,7}$

Macroscópicamente los tumores son de aspecto esferoidal o elípticos, bien circunscritos, compuestos de una cápsula con zonas hemorrágicas y necróticas en su interior en 50\% de los casos, la presencia de calcificaciones y septos muy friables se observa con menor frecuencia. ${ }^{4,5,7}$ La forma macroscópica de nuestro caso era quística, medía $8 \times 4.5 \times 3 \mathrm{~cm}$ con superficie lisa color rosado rojizo que eliminaba material sanguinolento y tejido degenerativo.

Microscópicamente son células uniformes de apariencia epiteloide, redondas u ovales, con núcleo central y cromatina fina sin actividad mitótica, el estroma es finamente vascularizado y se reconocen cristales de colesterol, histiocitos y calcificaciones; $;{ }^{4,5,7}$ el informe de nuestro estudio microscópico apoyó el diagnóstico de un carcinoma sólido papilar con presencia de embolias vasculares, compromiso perineural, presencia de calcificaciones dispersas y cambios quísticos degenerativos que necesitaron además técnica de inmunohistoquímica para corroborar el diagnóstico.

El tratamiento de elección es la resección quirúrgica, dependiendo la localización, se debe realizar pancreatoduodenectomía si la lesión se encuentra a nivel de la cabeza y cueIlo con compromiso duodenal; enucleación si es que existe compromiso transpancréatico y de localización central o pancreatectomía 
distal con esplenectomía en caso de encontrarse en la cola. El tratamiento quirúrgico presenta escasa mortalidad y bajas tasas de recidiva; ; $1,2,6$ en nuestro caso se decidió realizar enucleación de la masa tumoral con tutorización del conducto de Wirsung para asegurar su funcionalidad posterior a intervención quirúrgica.

Se ha afirmado que estos tumores son radiosensibles y en ocasiones se han tratado mediante quimioembolización, pero no está claro el papel de la radioterapia ni de la quimioterapia en su tratamiento. 3,6

Para diferenciarlos pueden ser de gran ayuda los métodos de inmunohistoquímica, los que se realizaron en nuestra paciente fueron: pancitoqueratina, la cual dio negativo en células neoplásicas, enolasa positivo débil en células neoplásicas, cromogranina negativa en células neoplásicas, HMB45 negativo en células neoplásicas, KI67 con índice de proliferación celular menor de 1\%. Estos tumores también presentan negatividad a marcadores endocrinos y marcadores neoplásicos como el CEA y el CA $19.9 ;^{7-9}$ en nuestra paciente no hubo evidencia de alteración en los mismos.

En general, los pacientes poseen un buen pronóstico y se reporta una supervivencia que

Figura 5:

Control

fistulográfico

normal con marcaje de vía biliar $y$

fuga de contraste hacia duodeno segunda porción.

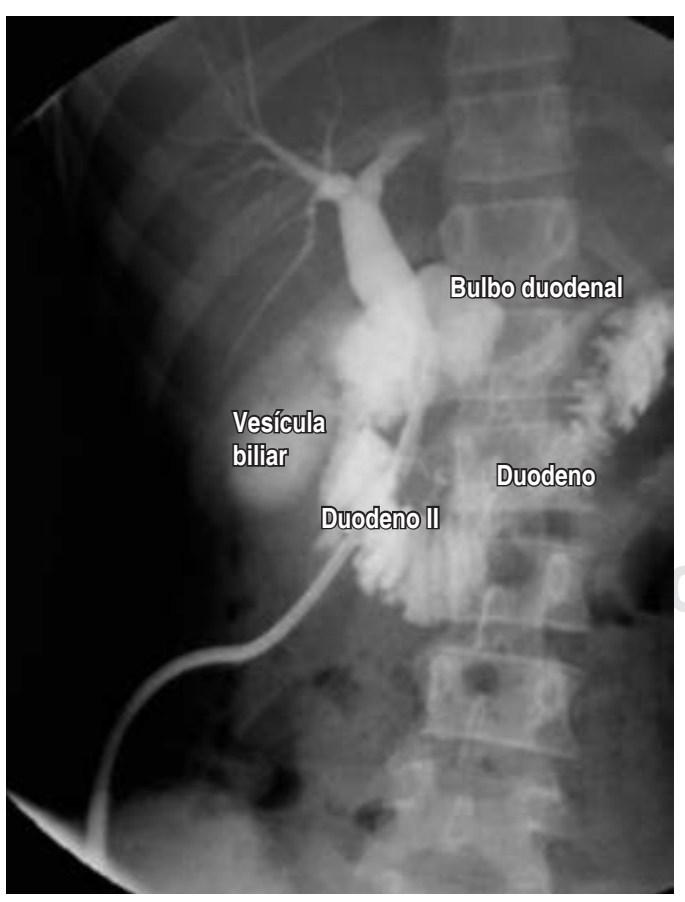

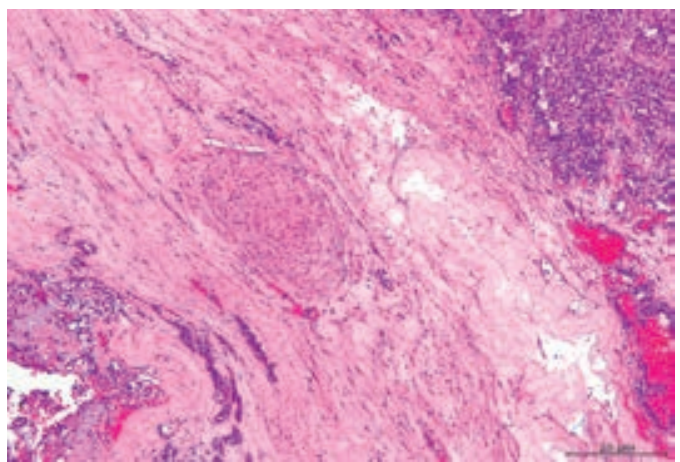

Figura 6: Microscopia con tinción de hematoxilinaeosina 20x, histopatología correspondiente a carcinoma sólido papilar pancreático, se evidencian embolias vasculares y compromiso perineural, calcificaciones dispersas y cambios quísticos degenerativos.

supera $90 \%$ a cinco años sólo con tratamiento quirúrgico. ${ }^{3,7,8,10}$ Nuestra paciente deberá ser sometida a seguimiento y control con exámenes de laboratorio e imágenes para descartar presencia de malignización posterior.

\section{CONCLUSIÓN}

El tumor de Frantz-Gruber es raro, diagnosticado comúnmente como hallazgo radiológico; posee características histopatológicas definidas, es de comportamiento benigno con bajas tasas de malignización; sin embargo, su tratamiento es quirúrgico para obtener un excelente pronóstico.

\section{REFERENCIAS}

1. Zumarán-Cuéllar O, Romero-Hernández T, BlancoBenavides R, Ramírez-Aceves R. Tumor de FrantzGruber: reporte de un caso. Revista Gastroenterología de México. 2004; 69: 236-239. Disponible en: http://www.imbiomed.com/1/1/articulos. php? method =showDetail\&id_articulo $=29453 \& i d$ seccion $=51 \&$ id_ejemplar $=3020 \&$ id_revista $=10$.

2. Vital-García H, González-Acosta M, Parra-Torres C. Tumor papilar sólido quístico de páncreas o tumor de Frantz-Gruber. Reporte de caso. Revista de Especialidades Médico-Quirúrgicas. 2015; 20: 100104. Disponible en: http://www.redalyc.org/articulo. oa?id=47345916017.

3. Álvarez-Pertuz H, Poblete-Otero F, Arana-Sáenz A, Hernández-Ramírez K, Villegas-Bermúdez D. Tumor de Frantz-Gruber, un tumor sólido pseudopapilar del páncreas poco frecuente. Acta Médica Costarricence. 2011; 53: 151-153. Disponible en: http://actamedica.medicos.cr/index. php/Acta_Medica/article/view/759. 
4. Sharma HP. Pseudopapillary tumor of pancreas. JK Science. 2011; 14: 199-201. Available in: http://www. jkscience.org/archive/volume134/Pseudopapillary\%20 Tumor\%20of\%20Pancreas.pdf.

5. Fernández JA. Tumores quísticos del páncreas: revisión de la literatura. Revista de Cirugía Española. 2003; 73: 297-308. Disponible en: http://www. elsevier.es/es-revista-cirugia-espanola-36-pdfS0009739X03721460.

6. Bacelar-Júnior E, Pereira GM, Torres $\mathrm{O}$, Santos L, Bezerra K, Rodrigues SA. Frantz's tumor of the pancreas. ABCD Arq Bras Cir Dig. 2010; 23: 212-213. Available in: http://www.scielo.br/pdf/abcd/v23n3/ v23n3a17.pdf.

7. Abad-Licham M, Sanchez-Lihon J, Celis-Zapata J. Tumor sólido pseudopapilar de páncreas en el Instituto Nacional de Enfermedades Neoplásicas. Rev Gastroenterol Perú. 2008; 28: 356-361. Disponible en http://www.scielo.org.pe/scielo.php?script=sci_ar ttext\&pid=S1022-51292008000400004.

8. Venturelli MF, Carrasco LC, Del Pozo LM, Cárcamo IC, Cárcamo IM, Venturelli LA. Tumor sólido-pseudopapilar del páncreas. Presentación de 3 casos clínicos. Cuad Cir. 2010; 24: 11-16. Disponible en: http://revistas.uach.cl/pdf/cuadcir/ v24n1/art02.pdf.

9. García HA, Monteferrario A, Pierini AL, Pierini L, Gatti D, Wenger M. Neoplasia sólida pseudopapilar del páncreas. Rev Argent Cirug. 2018; 110: 37-42. Disponible en: http://dx.doi.org/10.25132/raac.v110. n1.1319.es.

10. Mura R, Montiel A, Parquet G, Frachi R, Acosta L. Tumor sólido pseudopapilar del páncreas. Reporte de caso. Cir Parag. 2013; 37: 33-34. Disponible en: http://scielo.iics.una.py/pdf/sopaci/v37n2/ v37n2a09.pdf.

\section{Consideraciones y responsabilidad ética}

Protección de personas y animales. Los autores declaran que para esta investigación no se han realizado experimentos en seres humanos ni en animales.

Confidencialidad de los datos. Los autores declaran que han seguido los protocolos de su centro de trabajo sobre la publicación de datos de pacientes.

Derecho a la privacidad y consentimiento informado. Los autores han obtenido el consentimiento informado de los pacientes y/o sujetos referidos en el artículo. Este documento obra en poder del autor de correspondencia.

Financiamiento: Los autores declaran que no se obtuvo financiamiento de ninguna organización o institución para la realización de este artículo. Conflicto de intereses: Los autores declaran que no existe conflicto de intereses.

Correspondencia:

Dra. Danna Auria Aspiazu

E-mail: daria_221@hotmail.com 